\title{
Sequential Synthesis of 3d-3d'-4f Heterometallic Heptanuclear Clusters in between Lacunary Polyoxometalates
}

Rinta Sato, Kosuke Suzuki, Takuo Minato, Kazuya Yamaguchi, and Noritaka Mizuno*

Department of Applied Chemistry, School of Engineering, The University of Tokyo, 7-3-1

Hongo, Bunkyo-ku, Tokyo 113-8656, Japan.E-mail: tmizuno@mail.ecc.u-tokyo.ac.jp

Table S1. Selected BVS values for III $_{\mathrm{FeMn} 4 \mathrm{Gd} 2}$ and $\mathbf{I I I}_{\mathrm{FeMn} 4 \mathrm{Dy} 2}$

\begin{tabular}{|c|c|c|c|c|c|}
\hline & $\mathrm{III}_{\mathrm{FeMn4Gd2}}$ & III $_{\mathrm{FeMn4Dy2}}$ & & $\mathrm{III}_{\mathrm{FeMn4Gd2}}$ & III $_{\text {FeMn4Dy2 }}$ \\
\hline $\mathrm{Fe} 1$ & 2.83 & 2.90 & $\mathrm{O} 12$ & 1.98 & 1.87 \\
\hline $\mathrm{Mn} 1$ & 2.92 & 2.96 & $\mathrm{O} 13$ & 2.06 & 2.08 \\
\hline $\mathrm{Mn} 2$ & 3.02 & 2.96 & O14 & 2.08 & 2.06 \\
\hline $\operatorname{Ln} 1^{[\mathrm{a}]}$ & 2.97 & 3.00 & $\mathrm{O} 15$ & 1.93 & 1.98 \\
\hline W1 & 6.00 & 6.09 & O16 & 1.97 & 1.95 \\
\hline W2 & 6.07 & 6.00 & O17 & 1.94 & 1.99 \\
\hline W3 & 6.18 & 6.10 & O18 & 2.09 & 2.07 \\
\hline W4 & 6.08 & 5.95 & O19 & 1.99 & 1.97 \\
\hline W5 & 6.16 & 5.90 & $\mathrm{O} 20$ & 2.06 & 2.07 \\
\hline W6 & 6.36 & 6.25 & $\mathrm{O} 21$ & 2.03 & 1.99 \\
\hline W7 & 6.14 & 5.95 & $\mathrm{O} 22$ & 1.98 & 1.93 \\
\hline W8 & 6.04 & 5.96 & $\mathrm{O} 23$ & 2.03 & 2.06 \\
\hline W9 & 5.77 & 6.15 & $\mathrm{O} 24$ & 2.07 & 2.03 \\
\hline Sil & 4.23 & 4.02 & $\mathrm{O} 25$ & 1.94 & 1.93 \\
\hline $\mathrm{O} 1$ & 2.07 & 2.09 & O26 & 2.06 & 2.03 \\
\hline $\mathrm{O} 2$ & 2.10 & 2.09 & $\mathrm{O} 27$ & 2.04 & 2.08 \\
\hline $\mathrm{O} 3$ & 1.91 & 1.93 & $\mathrm{O} 28$ & 2.04 & 2.04 \\
\hline $\mathrm{O} 4$ & 1.97 & 1.95 & O29 & 1.90 & 1.86 \\
\hline O5 & 2.01 & 1.95 & O30 & 1.95 & 1.94 \\
\hline O6 & 1.85 & 1.92 & O31 & 2.00 & 2.00 \\
\hline O7 & 1.75 & 1.61 & O32 & 1.62 & 1.56 \\
\hline O8 & 1.67 & 1.64 & O33 & 1.58 & 1.67 \\
\hline O9 & 1.78 & 1.69 & O34 & 1.62 & 1.71 \\
\hline $\mathrm{O} 10$ & 1.88 & 1.71 & $\mathrm{O} 35$ & 1.96 & 1.95 \\
\hline O11 & 1.73 & 1.66 & & & \\
\hline
\end{tabular}


Table S2. Crystallographic data for $\mathbf{I I}_{\mathrm{FeCu}}$.

\begin{tabular}{ll}
\hline & \multicolumn{1}{c}{$\mathrm{II}_{\mathrm{FeCu} 4}$} \\
\hline formula & $\mathrm{C}_{124} \mathrm{Cl}_{12} \mathrm{Cu}_{4} \mathrm{FeN}_{7} \mathrm{O}_{72} \mathrm{Si}_{2} \mathrm{~W}_{18}$ \\
$F w\left(\mathrm{~g} \mathrm{~mol}^{-1}\right)$ & 6840.20 \\
cryst system & monoclinic \\
space group & $P 2_{1} / a(\# 14)$ \\
$a(\AA)$ & $19.2687(2)$ \\
$b(\AA)$ & $29.5016(3)$ \\
$c(\AA)$ & $19.3110(2)$ \\
$\beta(\mathrm{deg})$ & $110.7774(9)$ \\
$V\left(\AA^{3}\right)$ & $10263.56(19)$ \\
$Z$ & 2 \\
temp $(\mathrm{K})$ & $123(2)$ \\
$\rho_{\text {calcd }}\left(\mathrm{g} \mathrm{cm}{ }^{-3}\right)$ & 2.213 \\
$\mathrm{GOF}$ & 1.138 \\
$R_{1}[I>2 \sigma(I)]$ & 0.0909 \\
& $($ for 21778 data $)$ \\
$w R_{2}$ & 0.2404 \\
\hline
\end{tabular}


Table S3. Selected BVS values for $\mathbf{I I I}_{\mathrm{FeCu} 4 \mathrm{Gd} 2}$ and $\mathbf{I I I}_{\mathrm{FeCu} 4 \mathrm{Dy} \mathbf{2}}$

\begin{tabular}{|c|c|c|c|c|c|}
\hline & III $_{\mathrm{FeCu} 4 \mathrm{Gd} 2}$ & III $_{\mathrm{FeCu} 4 \mathrm{Dy} 2}$ & & III $_{\mathrm{FeCu} 4 \mathrm{Gd} 2}$ & III $_{\mathrm{FeCu} 4 \mathrm{Dy} 2}$ \\
\hline $\mathrm{Fe} 1$ & 2.84 & 2.90 & $\mathrm{O} 12$ & 1.77 & 1.76 \\
\hline $\mathrm{Cu} 1$ & 2.04 & 2.00 & O13 & 1.90 & 1.89 \\
\hline $\mathrm{Cu} 2$ & 2.00 & 2.04 & $\mathrm{O} 14$ & 2.07 & 2.09 \\
\hline $\operatorname{Ln} 1^{[\mathrm{a}]}$ & 3.13 & 3.09 & $\mathrm{O} 15$ & 2.05 & 2.04 \\
\hline W1 & 6.10 & 6.07 & O16 & 2.08 & 2.07 \\
\hline W2 & 6.10 & 6.13 & $\mathrm{O} 17$ & 2.04 & 2.09 \\
\hline W3 & 6.06 & 6.02 & O18 & 2.09 & 2.07 \\
\hline W4 & 6.06 & 6.05 & O19 & 1.88 & 1.89 \\
\hline W5 & 6.05 & 6.03 & $\mathrm{O} 20$ & 1.91 & 1.92 \\
\hline W6 & 5.99 & 6.03 & $\mathrm{O} 21$ & 1.93 & 1.92 \\
\hline W7 & 6.03 & 6.08 & $\mathrm{O} 22$ & 1.95 & 1.97 \\
\hline W8 & 5.94 & 6.11 & $\mathrm{O} 23$ & 2.02 & 2.04 \\
\hline W9 & 5.97 & 6.14 & $\mathrm{O} 24$ & 2.02 & 2.05 \\
\hline Si1 & 3.91 & 3.93 & $\mathrm{O} 25$ & 1.95 & 1.99 \\
\hline $\mathrm{O} 1$ & 2.03 & 1.98 & $\mathrm{O} 26$ & 2.09 & 2.05 \\
\hline $\mathrm{O} 2$ & 2.00 & 2.02 & $\mathrm{O} 27$ & 2.01 & 2.07 \\
\hline $\mathrm{O} 3$ & 1.55 & 1.29 & $\mathrm{O} 28$ & 1.97 & 1.98 \\
\hline $\mathrm{O} 4$ & 1.62 & 1.86 & O29 & 1.92 & 1.91 \\
\hline O5 & 1.84 & 1.67 & $\mathrm{O} 30$ & 1.97 & 2.02 \\
\hline O6 & 1.27 & 1.61 & O31 & 2.20 & 2.01 \\
\hline O7 & 1.74 & 1.75 & $\mathrm{O} 32$ & 1.73 & 1.74 \\
\hline O8 & 1.77 & 1.75 & $\mathrm{O} 33$ & 1.69 & 1.76 \\
\hline O9 & 1.71 & 1.77 & $\mathrm{O} 34$ & 1.77 & 1.77 \\
\hline O10 & 1.76 & 1.73 & $\mathrm{O} 35$ & 1.28 & 1.27 \\
\hline $\mathrm{O} 11$ & 1.71 & 1.75 & & & \\
\hline
\end{tabular}




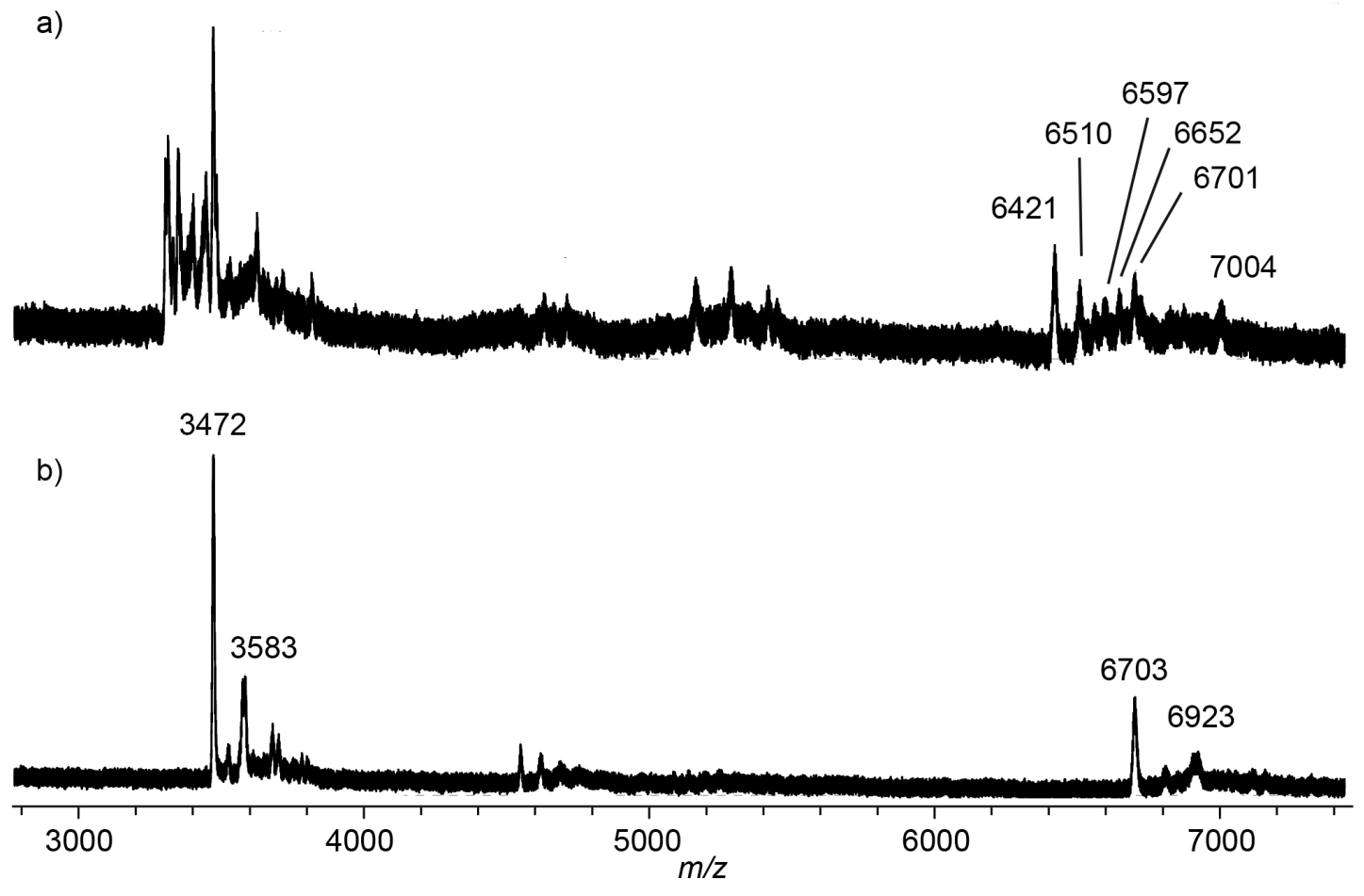

Figure S1. Positive ion CSI-mass spectra of the reaction solutions containing a) $\mathrm{Fe}(\mathrm{acac})_{3}, \mathrm{Mn}(\mathrm{acac})_{3}$, $\operatorname{Gd}(\mathrm{acac})_{3}$, and SiW9 (1:4:2:2 molar ratio) and b) $\mathrm{Mn}(\mathrm{acac})_{3}, \mathrm{Gd}(\mathrm{acac})_{3}$, and $\mathbf{I}_{\mathbf{F e}}$ (4:2:1 molar ratio) in 1,2-dichloroethane. The +1-signals in the range of $\mathrm{m} / \mathrm{z} 6000-7000$ were assignable to $\left[\mathrm{TBA}_{8} \mathrm{H}_{6} \mathrm{Mn}\left(\mathrm{SiW}_{9} \mathrm{O}_{33}\right)_{2}\right]^{+}\left(m / z \text { 6421), [TBA } \mathrm{T}_{7} \mathrm{Mn}_{2}\left(\mathrm{SiW}_{9} \mathrm{O}_{34}\right)_{2}\right]^{+}\left(\mathrm{m} / z \text { 6510), [ } \mathrm{TBA}_{8} \mathrm{H}_{8} \mathrm{Mn}_{3} \mathrm{O}_{2}\left(\mathrm{SiW}_{9} \mathrm{O}_{34}\right)_{2}\right]^{+}$ $\left(m / z\right.$ 6597), $\left[\mathrm{TBA}_{8} \mathrm{H}_{5} \mathrm{Mn}_{4} \mathrm{O}_{2}\left(\mathrm{SiW}_{9} \mathrm{O}_{34}\right)_{2}\right]^{+} \quad(m / z \quad 6652), \quad\left[\mathrm{TBA}_{8} \mathrm{H}_{2} \mathrm{Mn}_{5} \mathrm{O}_{2}\left(\mathrm{SiW}_{9} \mathrm{O}_{34}\right)_{2}\right]^{+}\left(\begin{array}{ll}\mathrm{m} / z & 6701\end{array}\right)$, $\left[\mathrm{TBA}_{8} \mathrm{H}_{4} \mathrm{Mn}_{4} \mathrm{Gd}(\mathrm{acac})_{2} \mathrm{O}_{2}\left(\mathrm{SiW}_{9} \mathrm{O}_{34}\right)_{2}\right]^{+} \quad\left(m / z\right.$ 7004), $\quad\left[\mathrm{TBA}_{8} \mathrm{H}_{2} \mathrm{FeMn}_{4} \mathrm{O}_{2}\left(\mathrm{SiW}_{9} \mathrm{O}_{34}\right)_{2}\right]^{+} \quad(\mathrm{m} / z \quad 6703)$, and $\left[\mathrm{TBA}_{8} \mathrm{H}_{5} \mathrm{FeMn}{ }_{3} \mathrm{Gd}(\mathrm{acac}) \mathrm{O}_{3}\left(\mathrm{SiW}_{9} \mathrm{O}_{34}\right)_{2}\right]^{+}(\mathrm{m} / z$ 6923). 


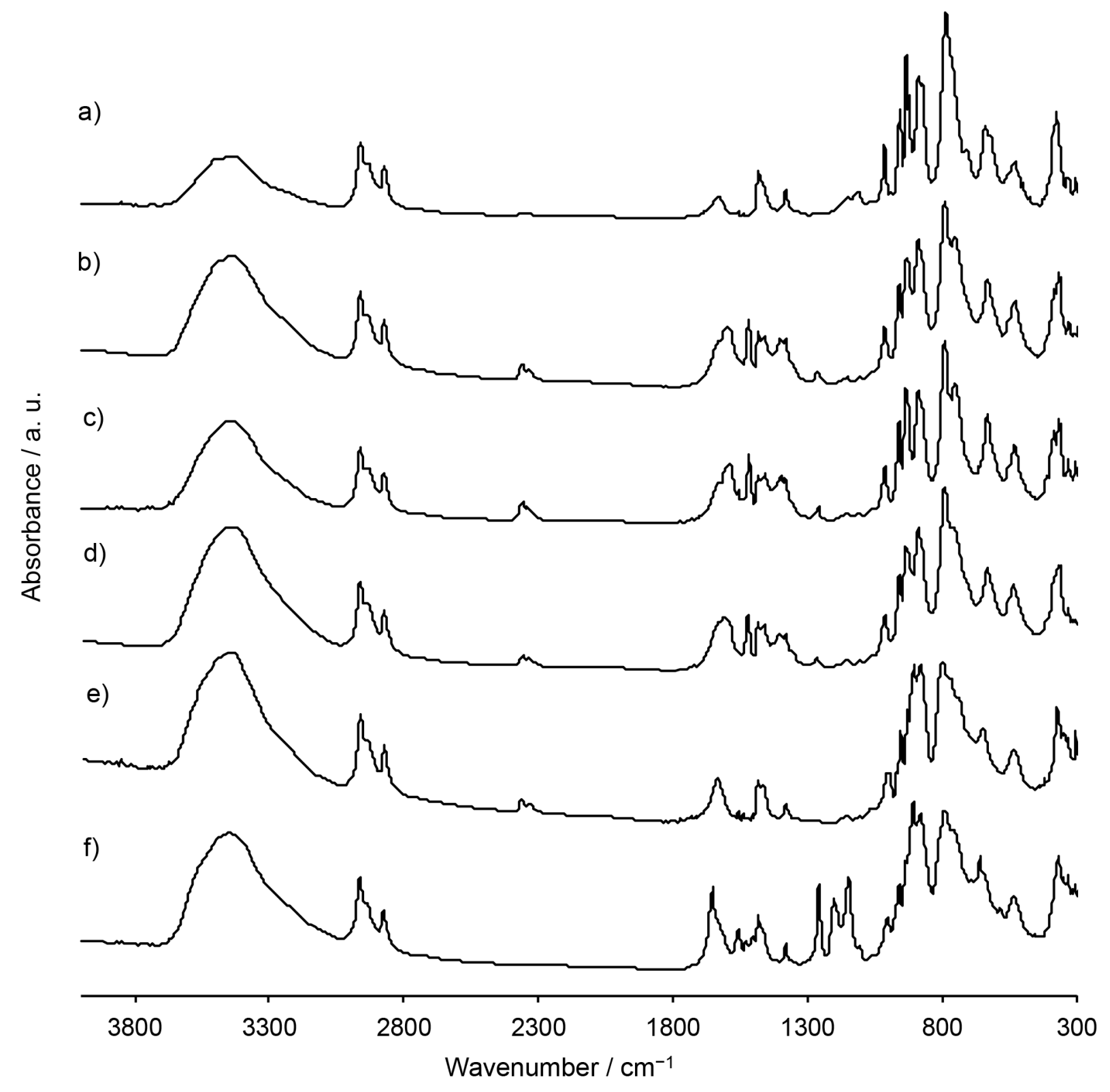

Figure S2. IR spectra of (a) $\mathbf{I I}_{\mathrm{FeMn} 4}$, (b) $\mathbf{I I I}_{\mathrm{FeMn} 4 \mathrm{Dy} 2}$, (c) $\mathbf{I I I} \mathbf{F e M n 4 G d 2}_{\mathrm{Fe}}$, (d) $\mathbf{I I I} \mathbf{F e M n 4 L u 2}_{\mathrm{Fe}}$, (e) $\mathbf{I I}_{\mathrm{FeCu}}$, and (f) III $_{\mathrm{FeCu} 4 \mathrm{Dy} 2}$. 

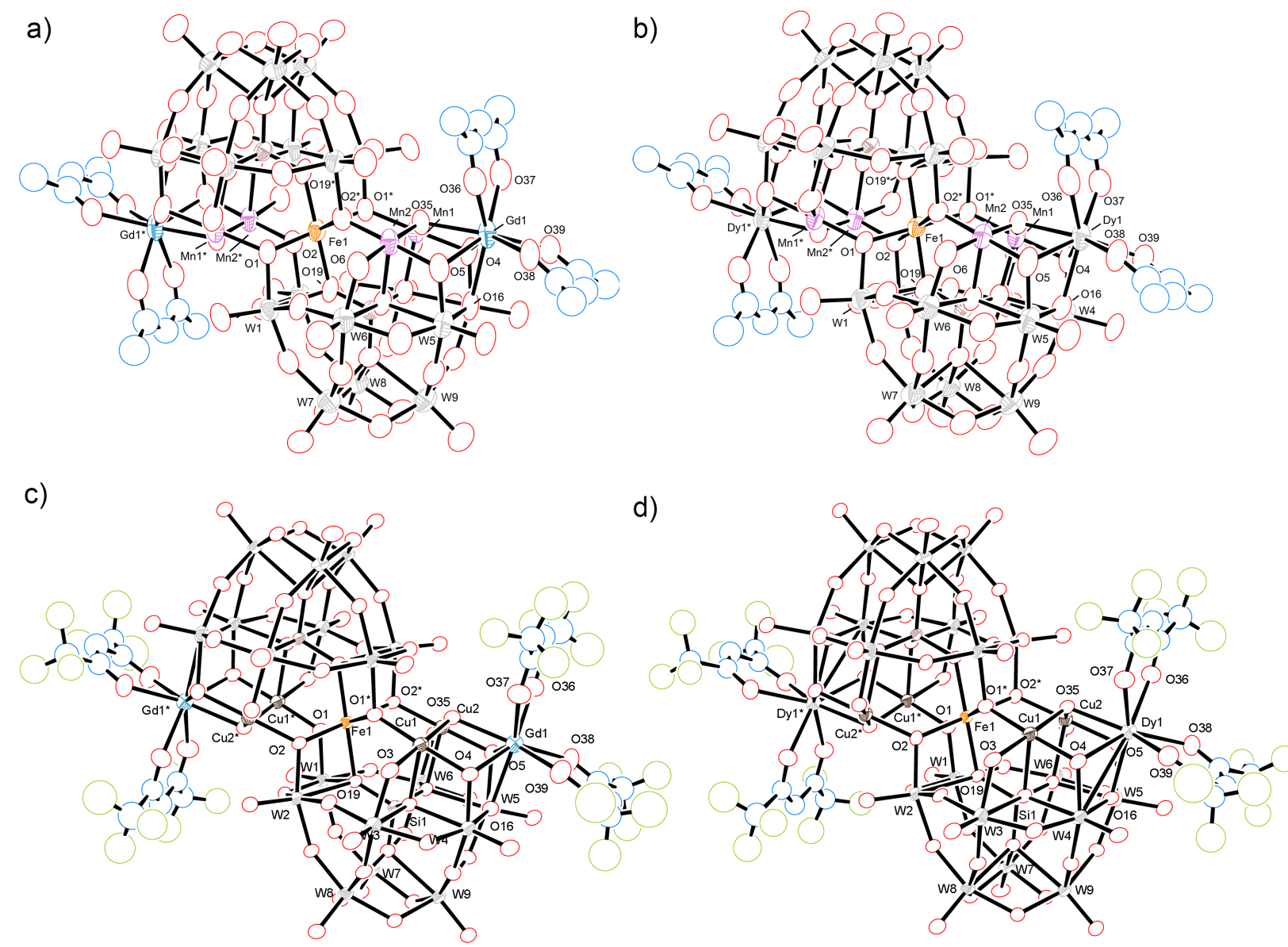

Figure S3. ORTEP representations of the anion parts of (a) $\mathbf{I I I}_{\mathrm{FeMn} 4 \mathbf{G d} 2}$, (b) $\mathbf{I I I}_{\mathrm{FeMn4Dy2}}$, (c) $\mathbf{I I I}_{\mathrm{FeCu} 4 \mathrm{Gd} 2}$, and (d) III $_{\text {FeCu4Dy2 } 2}$ with thermal ellipsoids drawn at the $50 \%$ probability level. 


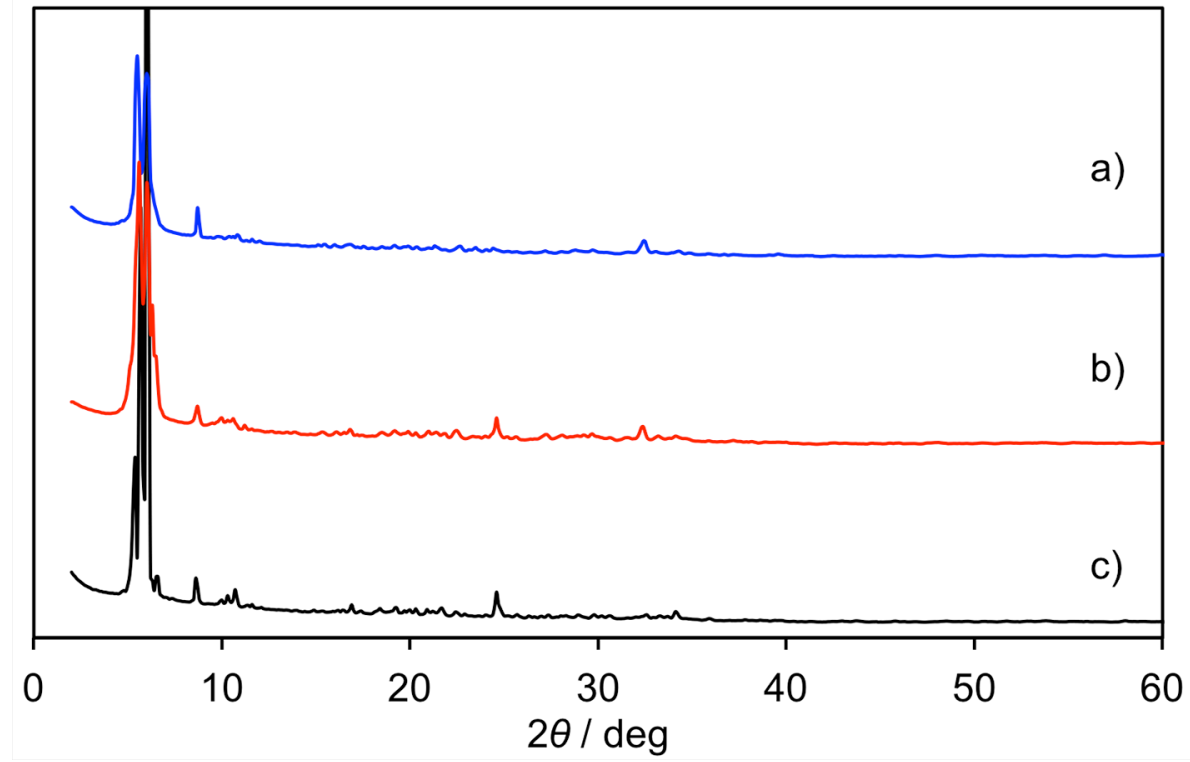

Figure S4. Powder X-ray diffraction patterns of (a) $\mathbf{I I I}_{\mathrm{FeMn} 4 \mathrm{Gd} 2}$, (b) $\mathbf{I I I}_{\mathrm{FeMn} 4 \mathrm{Dy} 2}$, and (c) $\mathbf{I I I}_{\mathrm{FeMn} 4 \mathrm{Lu} 2}$. 


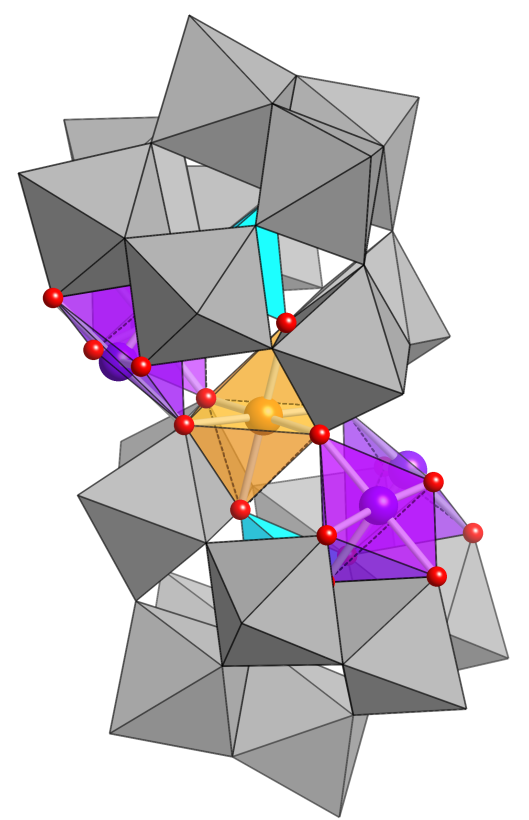

Figure S5. Polyhedral and ball-and-stick representation of the anion part of $\mathbf{I I}_{\mathbf{F e C u} 4}$. Orange, purple, gray, and light blue polyhedra represent $\left\{\mathrm{FeO}_{6}\right\},\left\{\mathrm{CuO}_{5}\right\},\left\{\mathrm{WO}_{6}\right\}$, and $\left\{\mathrm{SiO}_{4}\right\}$, respectively. 


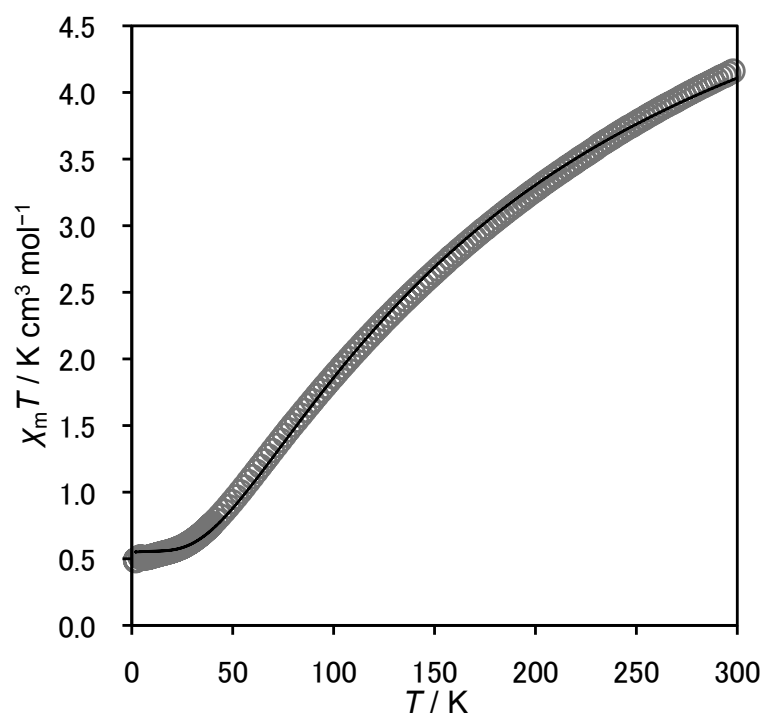

Figure S6. Temperature dependence of $\chi_{\mathrm{m}} T$ of $\mathbf{I I}_{\mathrm{FeCu}}$ under the applied field of $0.1 \mathrm{~T}$. Solid line represents the best fits adopting the Heisenberg-Dirac-Van Vleck Hamiltonian. 


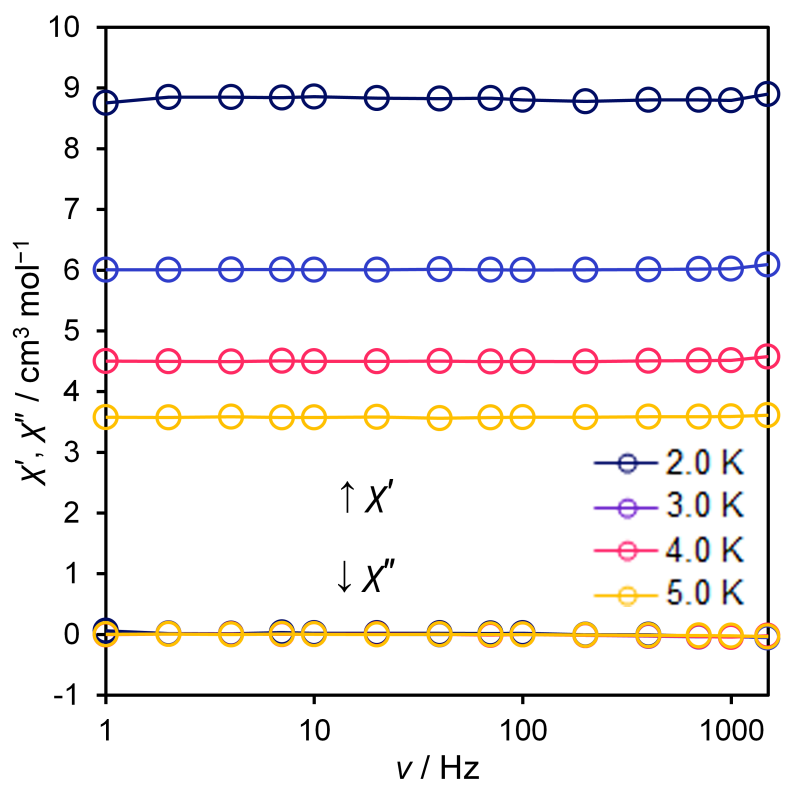

Figure S7. Frequency dependence of the ac magnetic susceptibility of $\mathbf{I I I}_{\mathbf{F e M n} 4 \mathbf{G d} \mathbf{2}}$ under zero applied dc field.

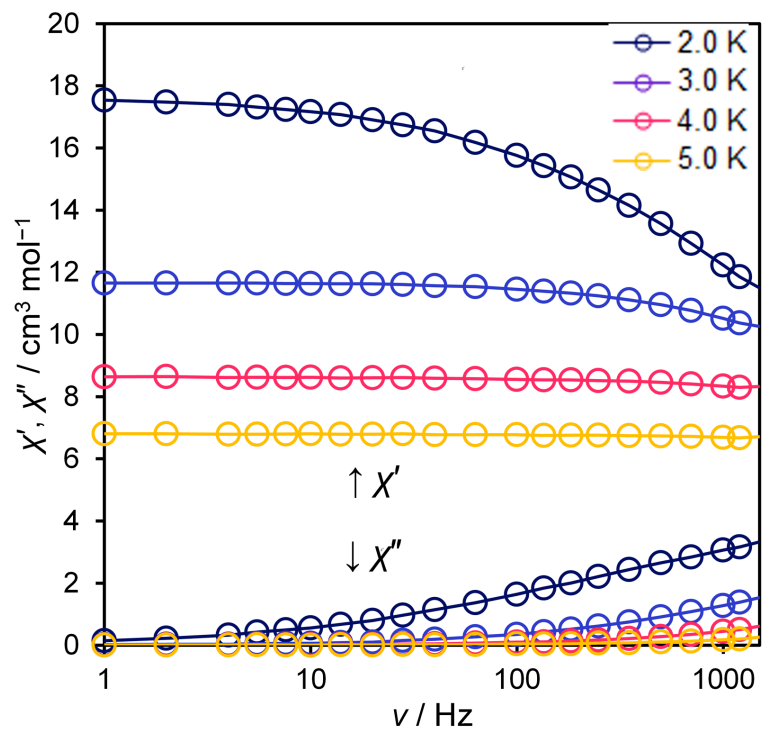

Figure S8. Frequency dependence of the ac magnetic susceptibility of $\mathbf{I I I}_{\mathbf{F e M n} 4 \mathbf{D y} \mathbf{2}}$ under zero applied dc field. 


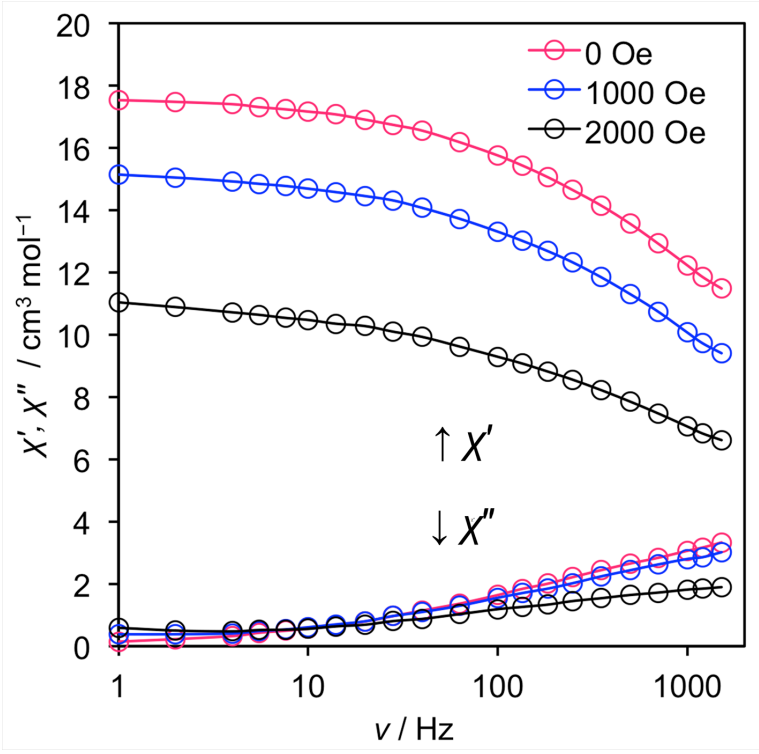

Figure S9. Frequency dependence of the ac magnetic susceptibility of $\mathbf{I I I}_{\mathbf{F e M n} 4 \mathbf{D y} 2}$ under various dc fields.

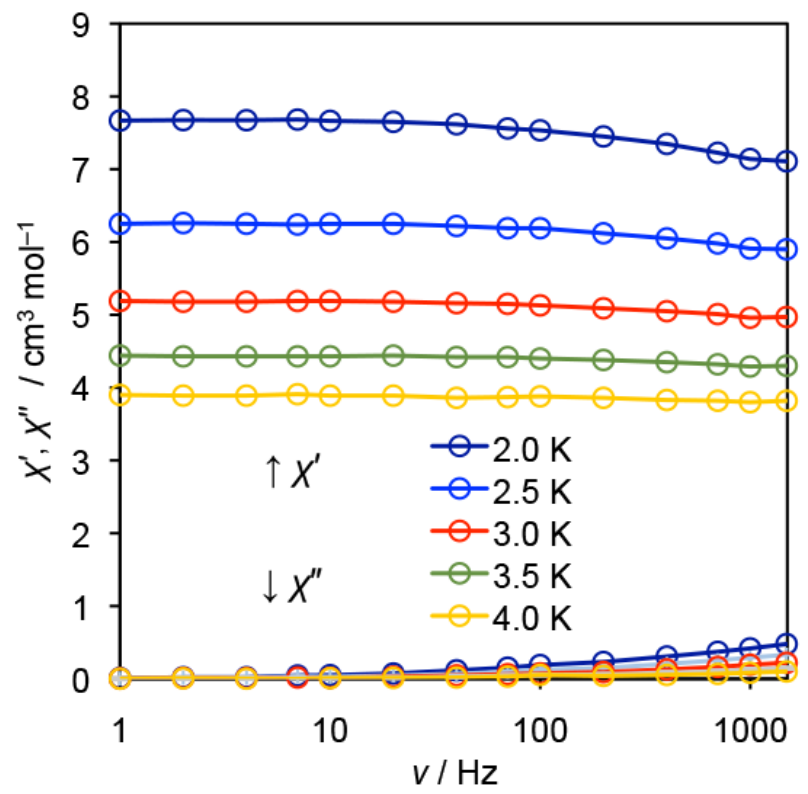

Figure S10. Frequency dependence of the ac magnetic susceptibility of $\mathbf{I I I}_{\mathbf{F e C u} 4 \mathbf{D y} 2}$ under zero applied dc field. 


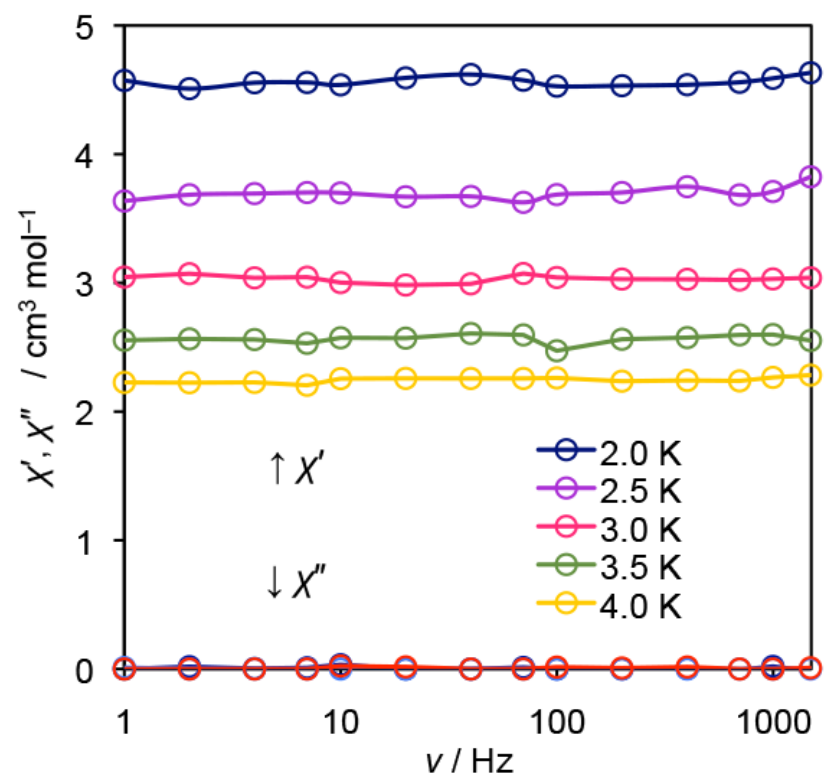

Figure S11. Frequency dependence of the ac magnetic susceptibility of $\mathbf{I I I}_{\mathrm{FeCu} 4 \mathrm{Gd} 2}$ under zero applied dc field. 